\title{
Talking to teenagers regarding sexual and reproductive health: Exploration of parents/guardians' views in Limpopo province, South Africa
}

\section{Matete E. Dilebo}

Limpopo College of Nursing - Sovenga Campus

Rachel T. Lebese

University of Venda School of Health Sciences

Dorah U. Ramathuba

University of Venda School of Health Sciences

LUFUNO MAKHADO ( $\square$ lufuno.makhado@univen.ac.za )

University of Venda https://orcid.org/0000-0003-1689-9308

\section{Research}

Keywords: discussion, sexual and reproductive health issues, teenagers, views of parents

Posted Date: February 18th, 2020

DOI: https://doi.org/10.21203/rs.2.23754/v1

License: (a) (i) This work is licensed under a Creative Commons Attribution 4.0 International License.

Read Full License 


\section{Abstract}

Background Sexual and reproductive health are sensitive topics that may not be easily discussed among different age groups in most cultures. Most parents are uncomfortable to communicate with their children about sexual and reproductive health. The rate of teenage pregnancy and sexually transmitted infections continues to rise as parents shift the responsibility of discussing sexual health to other members of the family or teachers. Many sexual and reproductive ill-health exists among teenagers as a result of poor communication with parents or elders. The aim of the study was to explore the views of parents/guardians regarding $t$ the discussion of sexual and reproductive health issues with teenagers.

Methods The study was conducted in Capricorn and Mopani Districts of Limpopo Province where teenage pregnancy rates were reported to be very high. A qualitative explanatory approach was used. The population comprised of parents/guardians to teenagers who were pregnant or having children. Data were collected through individual face to face interview guided by one central question. Data were analysed using Tesch's method. Measures to ensure trustworthiness and ethical issues were ensured.

Results Two themes emerged from the study: Views regarding discussing sexual and reproductive-related topics between parents and teenagers and Relationship of parents/guardians and their teenagers as an influence of discussions about SRH matters.

Conclusion Parents/guardians still view discussing sexual and reproductive health issues with teenagers as taboo and difficult. However, discussing sexual and reproductive health issues with teenagers is seen as a beneficial practise as it might influence them positively and may decrease unwanted sexual risks such as teenage pregnancies and HIV infections.

\section{Introduction}

Teenagers' face multiple challenges during their transition to adulthood. They are exposed to mixed messages that they receive from their peers, media and the internet and sometimes parents/guardians. More than often they fail to comprehend such information, leading to risky sexual behaviours such as unprotected sex, multiple sexual partnerships, and transactional which predispose young people to sexually transmitted infections, teenage pregnancy and HIV and AIDS [1]. Parents play a critical role in the growth, development and sexual socialization of their children and should be involved in the presentation of sex education and reproductive health. During Parent-child sexual communication, parents transmit sexual values, beliefs, information and expectations to their children with the aim of influencing sexual behaviours, attitudes and decision-making of their children [2].

Sexual and Reproductive Health (SRH) matters are sensitive issues to discuss with teenagers. Sociocultural and religious beliefs, as well as parents' educational level, are the most enhancing or inhibiting factors towards discussing such topics [3]. Furthermore, cultural beliefs and values dictate what parents 
should and should not say to their children about sexual health issues. In contrast, acculturation has also contributed to the sexual behaviours of adolescents. It was also indicated that acculturation is a dynamic and complex process by which individuals combine and meld their original and secondary cultures into a third, unique set of cultural values, practices, and beliefs [4]. The authors further reported that the less acculturated youths reported having sex at a lower rate, having few sexual partners and habitual condom use than their more acculturated peers.

Religion has also been cited as a factor that hinders parent-child communication, for example, the Protestant churches and Islams' believe in abstinence before marriage, maintaining of virginity and oppose sex education, and maintains that SRH programmes might encourage adolescents' preoccupation with the sex $[5,6]$. Religious beliefs had been contended to guide parents on what to discuss with their children, focusing on teaching their daughters about the virtues of virginity and that they should forget about sexual activities until they get married [7]. Hence this religious belief contributes to the hindrance that makes parents fail to communicate with their children about issues of sexual and reproductive health.

Parents in black African communities are uncomfortable in broaching the subject on reproductive sexual health and shifting this responsibility to the teachers at schools [3]. Furthermore, most male parents perceived talking about sexual health with children as shameful and immoral thus encouraging the children to engage in sexual intercourse [7, 8]. Similarly, parents do not talk to their children about SRH topics citing that it was culturally unacceptable [9]. Parents seemed to think that SRH-related issues concerned only sexual intercourse

Previous research showed that most of the communication between the parent/guardians and the teenagers focus on abstinence, menstruation, sex, HIV, contraception, unplanned pregnancy, and puberty and some of the content is not correct as parents have limited information about sexual health education $[2,10]$. The annual review of sex literature indicated that most of the mothers in the studies expressed relative comfort and willingness to discuss the consequences of sex, but not specific, fact-based information regarding intercourse and birth control [11]. The communication in most cases are unidirectional and takes the form of a lecture rather than dialogue and the consequences of sex were exaggerated, and what triggered the conversation was often the undesirable behaviour of the adolescents $[11,12]$. In addition, directive parents who tend to have a more authoritarian communication style do not invite open discussion and questions from children, few fathers provided explicit guidance, open and honest conversations which contributed to daughters' knowledge [11].

Limpopo province is experiencing the highest number of adolescent pregnancies, sexually transmitted infections and HIV and AIDS. The South African Broadcasting Cooperation (SABC) on the 16 October 2018 reported that 36 learners aged between 10 and 19 at one of the secondary schools in the Mulenzhe 
area, outside Thohoyandou were pregnant and children as young as nine, are sexually active, furthermore 13 of them being HIV positive [13]. This is an indication of absent parent-child communication which predispose adolescent learners to experiment with sex at an early age. For this reason, the purpose of this study was to explore the views of parents/guardians related to talking about sexual and reproductive health issues with teenagers.

Parent-child communication on sexual reproductive health has been identified as a protective factor for adolescent sexual and reproductive health, and interactive communication strategies include making sure adolescents' voices are heard to encourage an active exchange of questions and answers, assessing current knowledge and leaving room for future discussions [14].

\section{Research Design And Methods}

For the purpose of this research, a phenomenological qualitative exploratory descriptive design was used to explore the views of parents/guardians related to the talking about sexual and reproductive health issues with teenagers. A qualitative research approach was chosen because the study was conducted in the participant's homes which is a natural setting where human behaviour and events occur. This approach allowed the researcher to interview the participant and deepen the discussions by probing and making follow-ups on certain information. Observations were made during interviews for non-verbal cues to get the holistic nature of the problem under discussions. Field-notes were taken. Parental views were explored through probing, paraphrasing, and follow-up questions. The authors sought to pursue this study given the need to understand factors influencing the rise in teenage pregnancies, teenage HIV infection rate and termination of pregnancies.

The study was conducted in Capricorn and Mopani districts of Limpopo Province, South Africa, where the statistics of teenage pregnancies was the highest in the Province. The researchers identified, the high schools with the highest number of pregnant learners in each District and parents from the surrounding feeder villages to the school formed part of the study. Mavalana village in Mopani District which is a feeder village for Mavalana High School was used based on the SABC news report where 50 leaners in 2010 and 57 learners in 2011 were reported to be pregnant and Blood River village in Capricorn Districts which is the feeder village for Molautsi Secondary School with 27 leaners reported to be pregnant [13]. These two schools were the ones with the highest numbers of pregnant teenagers as reported on television.

The population consisted of both mothers and fathers including guardians of teenagers, who were pregnant or had babies in the current year of study. Non-probability purposive sampling method was used to select participants. Eighteen participants were selected until data saturation. Participants were identified through the school's life orientation teachers who had a list of leaners who were pregnant and 
those who had delivered. Appointments were first secured with the learners, parents after they had provided assent and informed consent was sought from identified teenagers. In families where both parents were available, they were interviewed separately to ensure confidentiality and privacy.

A central question was developed in English then translated into XiTsonga for parents at Mavalana Village and Sepedi for parents in Blood River Village to ensure understanding and getting in-depth information. The research idea was introduced to the parents during the initial group meeting and more information was shared when negotiating the signing of informed consent with individuals. A date for data collection was then set as rapport had been established. Data collection was conducted from May 2018 to October 2018. In families where both parents were available, they were interviewed separately to ensure confidentiality and privacy. Interviews were conducted in the participants' comfort of their homes. The individual face-to-face interview was used to collect data. An audiotape was also used to capture information. The interviews lasted for approximately 45 to 60 minutes. Field notes were written to capture verbal and non-verbal cues. A central question was posed in the same manner to each participant as follows: "What are your views regarding discussing sexual and reproductive health issues with your teenage child?"

Data were coded based on the themes and subthemes derived from the responses to the questions with the assistance of an independent coder. The recorded and documented data were compared. Data belonging to one group were assembled in one place to assist further analysis. Themes and sub-themes were formulated using Techs steps [15]. Trustworthiness was ensured through the four principles of credibility, confirmability and transferability. Credibility was ensured by a prolonged engagement which increased rapport and to clarify descriptions with participants through familiarity. Data triangulation was ensured by using different data collection methods through field notes, in-depth individual interviews, referential adequacy and co-coder. Confirmability was ensured by audit trail of voice recorder and the field notes to determine the conclusions, interpretations and recommendations if traced from their sources. Transferability was ensured through the thick description of the research methods and design.

\section{Results}

The participants' age ranged from 43 to 60 years with 6 males and 12 females. The participants had children ranging from 4-6 and of those children about 1-3 were pregnant or having a child (See Table 1).

\section{Insert Table 1 here}

Results from individual interviews yielded three themes which described the views of parents/guardians related to the discussion of sexual and reproductive health issues with teenagers (See Table 2). The themes incorporated religious beliefs affecting sexual reproductive health discussions with teenagers; communication and Discussion of sexual and reproductive health issues with teenagers; and parentteenager relationship enhances sexual reproductive health discussions which were substantiated by direct quotations from participants. 


\section{Insert Table 2 here}

Religious beliefs affecting sexual reproductive health discussions with teenagers.

Some parents, who were interviewed during the current study, disagreed with the introduction of SRH services in schools or parents being involved in discussing SRH with their children, indicating that it was against their religion, while others indicating that it was cultural taboo. In their own words the participants said:

"My religion prohibits such topics; no sex before marriage. We are trying to teach our children good morals, but you want to bring things that will promote immorality." (P12, Female, 50)

\section{Gender and cultural stereotypes influence SRH communication}

Gender and culture influence sexual communication compromising sexual health and the associated with doubts of seeking or providing reproductive health information. Gender-related issues were cited by some parents as factors causing discomfort. Male parents indicated that they could not talk to their daughters about menstruation, dating or contraception. These aspects are confirmed by the following statements from the interviewed parents:

"You know....in my culture it is a taboo to talk about sexual issues with children. When my 16-year-old girl fell pregnant, I blamed her mother for not talking to her. The same year my 18 years old son impregnated a 15-year-old. I was dumbfounded, didn't know whom to blame now. I thought it is her responsibility to talk to girls about issues like menstruation, contraception, pregnancy, but I failed too to talk to my son about protection." (P16, Male, 50)

\section{Communication and discussion of sexual and reproductive health issues with teenagers}

Parents expressed that it was difficult to discuss SRH issues with their own children or to answer their children's questions in this regard. Some parents indicated that they used warnings and threats to communicate with their children. Parents indicated that communication is usually triggered by instant circumstances occurring at a point in time.

"Hmm... it is sometimes difficult but sometimes we try, even though as a parent you feel ashamed to face your child and talk about SRH things" (P4, Female, 52)

"The only time I talk to my children about sex is when someone in the village died of HIV and AIDS, thereby warning them, other than that, I never talk to them." (P12, Female, 50) 
Some participants pointed out positive views that it was important to discuss SRH matters with teenagers so that they could be well informed and also understand the changes in their bodies. Some mothers said that their boys preferred to discuss SRH related matters with them rather than with their fathers. Other parents said that they were the ones who initiated these conversations during family meetings or during informal dialogues.

"Yes, I am open to my children and my children are able to tell me that there is 1, 2, 3 and most of the time I am with them. I always tell them, hey, you people, especially the boys, you play around too much, you must use condoms because there are many diseases." (P3, Female, 43)

"Since my experience with teenage pregnancy, I vowed that I don't want any of my children to go through what I went through. I always share with them my story and tell them what to do to avoid that. I think they listened because to date none of them is a victim of teenage pregnancy. My firstborn had a child at 24 and the second born at 26. The last born is 23 and does not have a child. So, talking to children is important." (P7, Male, 60)

"When my girl started menstruating, she was afraid of me, but I sat down with her and said look, I know you are afraid of me as your mother. There is no one who can help you. You must tell me step-by-step what is happening, and I started explaining. Even the boys when they come to me, they come freely, I tell them and say look, let me tell you when you play in the blankets, a baby will come, one, two children. You will end up unable to buy something for yourself because you will be maintaining all these children. I even teach them that every action has consequences which are bad. I am very free to talk to them; I don't have a problem. They also come to me when they have a problem. They don't go to their father (laughing), they come to me. They will say mum [mother] because you work at the hospital what do you see there. Then I explain to them and say to them, this and this is not good, things like Metsosha [sexual stimulants], those things are not good. Leave everything to nature as determined by God." (P11, Female, 52)

\section{Parent-teenager relationship enhances sexual reproductive health discussions}

Parents interviewed during the current study indicated that they had good relationships with their teenage children, which made it easier to engage in communication about SRH-related topics with them. However, 
some parents did not feel comfortable to engage in SRH-related discussions with their teenage children. Some parents indicated that it was easy for them to discuss SRH matters with their teenage children. Both male and female teenagers were more comfortable discussing SRH matters with their mothers than with their fathers. In their own words, the participants said:

"Fortunately, I and my little girl are friends, she is able to [can] share, and I am also able to guide her. It is just like touch-ups. When talking to her I can see that she knows a little bit of something." (P 13, Male, 56)

"Yes, I as a parent have established a good relationship with my child; we are free to talk anytime. I am able to communicate with him, guide him, give him information about life so that he can know and understand that if he has a girlfriend at that age he will fall into trouble." (P9, Female, 47)

Some teenagers' parents, who participated in the current study, indicated that they had good relationships with their children. This enhanced their communication about SRH matters. Female participants reflected that their teenage sons sought advice, related to SRH issues from them rather than from their fathers. This was indicated as follows:

"I am able to communicate with my son, guide him, give him information about life so that he can know and understand that if he can have a girlfriend at that age he will fall into trouble, the trouble of impregnating a girl or contract diseases" (P18, Female, 47)

"I tell them that if they want to start doing such things, they should use disease prevention measures, like hmm... condom. A girl must at least use a condom as well and contraceptives to prevent STIs, HIV and AIDS as well as pregnancy" (P1, Female, 51)

The participants indicated that having a close relationship with their teenage children made it possible for them to be aware of dangerous situations faced by teenagers. The participants also said in some instances that teenagers could not talk about difficult situations, but having close relationships, enabled them to be open. Parents indicated that it is important to have good relationships with their teenage children as this enhanced teenagers' openness to reveal hidden issues that in actual instances they could not talk about if parents were unapproachable. The current study revealed that some parents could identify situations which affected teenagers and could give support and advice. This was found to be 
useful to teenagers because their challenges related to SRH were addressed. The following relevant statements from the participants support this finding:

"I teach my teenage girl that she should not allow boys to touch her and that she should run away from older men who take advantage of young girls; they want to ruin their future [sic]. Actually, my girl was telling me that when they come back from school men will stop their cars and call them" (P2, Female, 55)

Another participant said:

"Because we don't know what happens when they get out of the gates and go to school, what kind of friends do they meet, what is it that they tell them what we don't tell them. So, I just keep on telling them this sexual and reproductive health issues for them to be able to protect themselves so that even if they are to engage in sexual relationships, they must protect themselves, they must start engaging knowingly, they must not only protect themselves but knowingly" (P17, Male, 57)

\section{Discussion Of Findings}

The findings of the study indicate that parents because of religious affiliation or adherence to cultural practices were negative towards discussing of SRH with their children. Similarly, Catholic schools and parents followed religious teachings with regard to sexual and reproductive education which emphasize abstinence [5]. In support, in the African culture, it would be considered a taboo to talk about sex with young adults or adolescents [16].

Some religious beliefs do not support the discussion of SRH issues with children, arguing that it would promote immorality. However, some parents realized that there was a need for these services to be provided in schools considering the high teenage pregnancy rate. It has been noted that most of the participants place more emphasis on sexual intercourse and ignore other aspects related to SRH. It is therefore important to assist communities and school in attending to all aspects that form part of the $\mathrm{SRH}$.

According to participants discussing issues related to $\mathrm{SRH}$ was viewed as being very difficult. Use of threats and indirect language was said to be some of the strategies used to initiate a discussion. In support, parents played a relatively small role in sexual socialization and education of adolescents, which could contribute to adolescents' engagement in sexual intercourse without proper guidance [17]. Others study reiterated that parents were very uncomfortable to talk about or discuss sex and sexuality issues 
with their children and shifting the responsibility to teachers and nurses $[2,18]$. Such poor communication between parents and teenagers could contribute to high rates of teenage pregnancies, STIs, HIV and AIDS infections. Parents might talk indirectly about sex with their children, something that could lead to misinterpretations and distortions of the essence of their sex education messages [19]. Furthermore, most parents felt that talking about sex with one's children was unpleasant, hence they had to wait for clues or triggers that a child was sexually active before they warned and threatened about the consequences of engaging in sex.

Factors which contributed towards making it difficult for parents to discuss SRH issues with their children were found to be ignorance, culture, gender, fear, age difference, and outsourcing of responsibility to teachers or health care professionals. Although parents could not communicate about SRH issues with their children, they showed concern about their children's safety as far as issues of sexuality were concerned. The parents reported that some of their teenage children obtained information from social media without parental guidance, which could have negative consequences. It is necessary that parents be given necessary support related to how they can engage teenagers and resources necessary to use as a reference where there is a lack of information.

The participants in the present study revealed that they realized the importance of discussing SRH issues with their teenage children. Mothers were found to be the ones that communicate more with their teenage children regarding SRH. Participants were concerned about their children's safety and about the consequences of unprotected sexual engagements. Similarly, some parents did educate their adolescents about sex, STIs and HIV [3]. Parents also encouraged their adolescent children to have one sex partner and always to use protection when engaging in sexual activities. Communication about SRH had sometimes been initiated by parents [20].

Parent-child SRH communication had been found to be mainly delivered by mothers and rarely by fathers [21]. Most young people are more relaxed talking to their mothers than talking to their fathers, but some adolescents do not trust their parents and censored what they could tell their parents for fear of punishment [22]. It has however been noted that the absence of the fathers in the discussions could have a negative effect on the outcome of such discussions. Involvement of both parents in the discussions could have a positive outcome as teenagers could realise the importance of the subject by the involvement of both parents.

The findings show that some parents had good relationships with their teenage children which facilitated two-way communication, but mothers seemed to communicate more often with their teenage children 
than fathers. Researchers also supported the findings that parent-child communication about SRH happened in most families and the communication was mainly on the same-sex basis, mother-daughter and rarely father-son or father-daughter [22]. On the contrary, a study revealed that both Australian fathers and mothers encouraged their children to protect themselves and others when engaging in sexual activities [23]. Of noteworthy, mother-child closeness is related to the later onset of sexual intercourse for daughters, but not for sons [24]. These findings appear to be contrary to a study that indicated that there was minimal if any, dialogue about sexual health between teenagers and their parents [25]. Thus, it is important to promote, encourage and maintain parent-child relationship s well as the dialogues related to sexual and reproductive health.

A positive relationship between parents and their teenage children was said to open a good communication channel between parents and teenagers. Similar findings to those of the current study demonstrated that the most consistent finding of numerous studies was that parent-child connectedness (support, closeness, and warmth) was related to lower adolescent pregnancy risk attributed to delayed and reduced frequency of sexual intercourse [25].

It has been identified as a close relationship between parents and teenagers open a door for parents to realise difficulties that face teenagers which they themselves could not have faced. This allows them to walk with them in this path and understand the world of the teenagers to can intervene appropriately. Contrary to these findings, other authors found that families rarely discussed contraception and condoms with their teenagers [23]. This study, however, did not indicate if this absence of communication was related to a strained relationship.

\section{Limitations And Strengths}

The study was conducted in Capricorn and Mopani districts of Limpopo Province and therefore, the results cannot be generalized to other districts without conducting similar studies elsewhere. The strength of the study was individual interviews which were used to obtain in-depth information.

\section{Conclusion}

The study highlighted that parents have trouble discussing sexual and reproductive health issues with their teenage children. Therefore, workshops to educate parents are recommended. The study focused on views of parents related to discussing sexual and reproductive health issues with teenagers. The findings yielded positive and negative responses as some parents were comfortable, while others displayed some discomfort. Parents should be empowered to be able to communicate sexual and reproductive issues in a frank and assertive manner. Breaking cultural, religious and gender stereotypes and strengthen their knowledge regarding sexual health issues. 


\section{Declarations}

Acknowledgements

The authors acknowledge all parents/guardians who participated in the study.

\section{Funding}

The study was funded by the University of Venda Research and Publication Committee (RPC)

\section{Contributions}

MED, RTL and DUR conceptualized and designed the study. MED conducted (Data collection) the study. LM, MDM, RTL and SMM analysed data, wrote, read, edited and approved the final manuscript.

\section{Ethics approval and consent to participate}

Permission to conduct the study was obtained from the University of Venda Ethics Committee (SHS/15/PH/19/0110) and the Department of Health and the Department of Education, Limpopo Province. Permission was also be sought from the participating Health Facilities where Health Care workers would be interviewed. Fundamental ethical principles were adhered to throughout the study. The participants' rights were explained. Informed written consent was sought from the participants prior to commencement. Participation in the study was voluntary and confidentiality and data safety were maintained.

\section{Consent for publication}

Not applicable.

\section{Availability of data and materials}

The datasets generated and/or analyzed during the current study are not publicly available due to the nature of ethical approval which stated that only the research team will have access to the collected data but are available from the corresponding author on reasonable request.

\section{Competing interests}

The authors declare that they have no competing interests.

\section{Abbreviations}

SRH-Sexual and Reproductive Health

HIV- Human Immunodeficiency Virus 
AIDS- Acquired Immunodeficiency Syndrome

STI- Sexually Transmitted Infections

\section{References}

1. Vickery, J.R., 2017. Worried about the wrong things: Youth, risk, and opportunity in the digital world. MIT Press

2. Manu AA, Mba CJ, Asare GQ, Odoi-Agyarko K, Asante RK. Parent-child communication about sexual and reproductive health: evidence from the Brong Ahafo Region, Ghana. Reproductive Health. 2015;12(1):16.

3. Phiri CM, Lebese RT, Maputle MS, Parents' perceptions regarding sex education of their adolescent children: Perspectives from a community in Mopani District, South Africa. African Journal for Physical, Health Education, Recreation and Dance (AJPHERD) Supplement, 2013;1(1):12-20.

4. McCullough Cosgrove J, LeCroy CW, Fordney M, Voelkel D. Considering the role of acculturation in parent-child communication about sexual health. 2018: S13-S14.

5. Wray A, Ussher JM, Perz J. Constructions and experiences of sexual health among young, heterosexual, unmarried Muslim women immigrants in Australia. Culture, health \& sexuality. 2014;16(1):76-89.

6. DiCenso A, Guyatt G, Willan A, Griffith L. Interventions to reduce unintended pregnancies among adolescents: a systematic review of randomised controlled trials. BMJ. 2002;324(7351):1426.

7. Motsomi K, Makanjee C, Basera T, Nyasulu P. Factors affecting effective communication about sexual and reproductive health issues between parents and adolescents in Zandspruit informal settlement, Johannesburg, South Africa. The Pan African Medical Journal. 2016;25.

8. Muhwezi WW, Katahoire AR, Banura C, Mugooda H, Kwesiga D, Bastien S, Klepp KI. Perceptions and experiences of adolescents, parents and school administrators regarding adolescent-parent communication on sexual and reproductive health issues in urban and rural Uganda. Reproductive health. 2015;12(1):110.

9. Kluge J. Sex education in adolescents-an opportunity for HIV and pregnancy prevention: female adolescent health. Professional Nursing Today. 2006 Jan 1;10(1):24-7.

10. Kajula LJ, Sheon N, De Vries H, Kaaya SF, Aarø LE. Dynamics of parent-adolescent communication on sexual health and HIV/AIDS in Tanzania. AIDS and Behavior. 2014;18(1):69-74.

11. Flores D, Barroso J. 21 st century parent-child sex communication in the United States: A process review. The Journal of Sex Research. 2017;54(4-5):532-48. 
12. Mabunda A, Madiba S. The context of parent-child communication about sexuality and HIV prevention; the perspectives of high school learners in Gauteng province, South Africa. Pula: Botswana Journal of African Studies. 2017;31:162-74.

13. SABC News, 2016: on line

14. Edwards LL, Reis JS. A Five-Step Process for Interactive Parent-Adolescent Communication About HIV Prevention: Advice from Parents Living With HIV/AIDS. J HIV AIDS Soc Serv. 2014;13(1):59-78. DOI: 10.1080/15381501.2013.775686.

15. Creswell JW, Creswell JD. Research design: Qualitative, quantitative, and mixed methods approach. Sage publications; 2017.

16. Baloyi ME. The "vat-en-sit" unions as a threat to the stability of African marriage in South Africa: African theological pastoral perspective. Phronimon. 2016;17(2):1-6.

17. Kunkel D, Farrar KM, Eyal K, Biely E, Donnerstein E, Rideout V. Sexual socialization messages on entertainment television: Comparing content trends 1997-2002. Media Psychology. 2007;9(3):595622.

18. Tsakani LR, Davhana-Maselesele M, Obi LC. Teenagers' experiences of sexual health dialogue in the rural villages of the Vhembe District, Limpopo Province. Health SA Gesondheid. 2011;16(1).

19. Wight $D$, Williamson $L$, Henderson M. Parental influences on young people's sexual behaviour: $A$ longitudinal analysis. Journal of adolescence. 2006;29(4):473-94.

20. Shier D, Butler J, Lewis R. Hole's essentials of human anatomy \& physiology. New York: McGraw-Hill Education; 2015.

21. McNeely C, Shew ML, Beuhring T, Sieving R, Miller BC, Blum RW. Mothers' influence on the timing of first sex among 14-and 15-year-olds. Journal of adolescent health. 2002;31(3):256-65.

22. Wamoyi J, Fenwick A, Urassa M, Zaba B, Stones W. Parent-child communication about sexual and reproductive health in rural Tanzania: Implications for young people's sexual health interventions. Reproductive Health. 2010;7(1):6.

23. Berne LA, Patton W, Milton J, Hunt LY, Wright S, Peppard J, Dodd J. A qualitative assessment of Australian parents' perceptions of sexuality education and communication. Journal of Sex Education and therapy. 2000;25(2-3):161-8.

24. Miller BC, Benson B, Galbraith KA. Family relationships and adolescent pregnancy risk: A research synthesis. Developmental review. 2001;21(1):1-38.

25. Lebese RT, Maputle MS, Mabunda JT, Chauke PK. Knowledge, attitudes and perception of students on teenage pregnancy: a case study of rural-based University students in South Africa. Journal of Human Ecology. 2015;51(1-2):55-65.

\section{Tables}


Table 1: Characteristics of the participants in the two villages.

\begin{tabular}{ccccc}
\hline Village & Gender & Age & \# of children & \# of children pregnant or having a child \\
\hline P1 & F & 51 & 4 & 1 \\
\hline P2 & F & 55 & 6 & 2 \\
\hline P3 & F & 43 & 4 & 2 \\
\hline P4 & F & 52 & 4 & 1 \\
\hline P4 & M & 58 & 5 & 1 \\
\hline P5 & F & 55 & 4 & 1 \\
\hline P6 & F & 56 & 4 & 1 \\
\hline P7 & M & 60 & 6 & 3 \\
\hline P8 & M & 56 & 5 & 2 \\
\hline P9 & F & 47 & 4 & 2 \\
\hline P10 & F & 50 & 4 & 1 \\
\hline P11 & M & 52 & 4 & 1 \\
\hline P12 & F & 50 & 4 & 1 \\
\hline P13 & M & 56 & 5 & 2 \\
\hline P14 & F & 53 & 4 & 2 \\
\hline P15 & F & 50 & 6 & 2 \\
\hline P16 & F & 50 & 5 & 1 \\
\hline P17 & M & 57 & 4 & 1 \\
\hline P18 & F & 47 & 4 & 1 \\
\hline & & & &
\end{tabular}

Key: P - Participant, F - Female, $M$ - Male, \#- Number

Table 2: Themes on views of parents/guardians about the discussion of SRH issues with teenagers.

1. Religious beliefs affecting sexual and reproductive health discussions with teenagers

1. Gender and cultural stereotypes influence SRH communication

1. Communication and Discussion of sexual and reproductive health issues with teenagers

1. Parent-teenager relationship enhances sexual and reproductive health discussions 